\title{
K2-140b - an eccentric 6.57 d transiting hot Jupiter in Virgo
}

\author{
H. A. C. Giles, ${ }^{1 \star}$ D. Bayliss, ${ }^{1}$ N. Espinoza, ${ }^{2,3}$ R. Brahm, ${ }^{2,3}$ S. Blanco-Cuaresma,,${ }^{1,4}$ \\ A. Shporer, ${ }^{5}$ D. Armstrong, ${ }^{6}$ C. Lovis,${ }^{1}$ S. Udry, ${ }^{1}$ F. Bouchy, ${ }^{1}$ M. Marmier, ${ }^{1}$
} A. Jordán, ${ }^{2,3,7}$ J. Bento, ${ }^{8}$ A. Collier Cameron, ${ }^{9}$ R. Sefako, ${ }^{10}$ W. D. Cochran, ${ }^{11}$ F. Rojas, ${ }^{2}$ M. Rabus, ${ }^{2}$ J. S. Jenkins, ${ }^{12}$ M. Jones, ${ }^{13}$ B. Pantoja, ${ }^{12,13}$ M. Soto, ${ }^{12}$ R. Jensen-Clem, ${ }^{14}$ D.A. Duev, ${ }^{14}$ M. Salama, ${ }^{15}$ R. Riddle,${ }^{14}$ C. Baranec ${ }^{15}$ and N. M. Law ${ }^{16}$

${ }_{1}^{1}$ Observatoire de Genève, Université de Genève, Chemin des Maillettes 51, CH-1290 Versoix, Switzerland

${ }^{2}$ Instituto de Astrofísica, Facultad de Física, Pontificia Universidad Católica de Chile, Av. Vicuña Mackenna 4860, 782-0436 Macul, Santiago, Chile

${ }^{3}$ Millennium Institute of Astrophysics, Av. Vicuña Mackenna 4860, 782-0436 Macul, Santiago, Chile

${ }^{4}$ Harvard-Smithsonian Center for Astrophysics, 60 Garden Street, Cambridge, MA 02138, USA

${ }^{5}$ Division of Geological and Planetary Sciences, California Institute of Technology, Pasadena, CA 91125, USA

${ }^{6}$ Department of Physics, University of Warwick, Gibbet Hill Road, Coventry CV4 7AL, UK

${ }^{7}$ Max-Planck-Institut für Astronomie, Königstuhl 17, D-69117 Heidelberg, Germany

${ }^{8}$ Research School of Astronomy and Astrophysics, Mount Stromlo Observatory, Australian National University, Weston, ACT 2611, Australia

${ }^{9}$ Centre for Exoplanet Science, SUPA School of Physics and Astronomy, University of St Andrews, North Haugh, St Andrews KY16 9SS, UK

${ }^{10}$ South African Astronomical Observatory, PO Box 9, Observatory 7935, South Africa

${ }^{11}$ McDonald Observatory and Department of Astronomy, University of Texas at Austin, Austin, TX 78712, USA

${ }^{12}$ Departamento de Astronomía, Universidad de Chile, Casilla 36-D, 7591245 Santiago, Chile

${ }^{13}$ European Southern Observatory, Alonso de Cordova 3107, Vitacura, Casilla 19001, Santiago, Chile

${ }^{14}$ Department of Astronomy, California Institute of Technology, 1200 E. California Blvd., Pasadena, CA 91101, USA

${ }^{15}$ Institute for Astronomy, University of Hawai ‘i at Mānoa, Hilo, HI 96720-2700, USA

${ }^{16}$ Department of Physics and Astronomy, University of North Carolina at Chapel Hill, Chapel Hill, NC 27599-3255, USA

\begin{abstract}
We present the discovery of K2-140b, a $P=6.57 \mathrm{~d}$ Jupiter-mass $\left(M_{P}=1.019 \pm 0.070 \mathrm{M}_{\mathrm{Jup}}\right)$ planet transiting a $\mathrm{V}=12.5$ (G5-spectral type) star in an eccentric orbit $\left(\mathrm{e}=0.120_{-0.046}^{+0.056}\right)$ detected using a combination of $K 2$ photometry and ground-based observations. With a radius of $1.095 \pm 0.018 \mathrm{R}_{\text {Jup }}$, the planet has a bulk density of $0.726 \pm 0.062 \rho_{\text {Jup }}$. The host star has a $[\mathrm{Fe} / \mathrm{H}]$ of $0.12 \pm 0.045$, and from the $K 2$ light curve, we find a rotation period for the star of $16.3 \pm 0.1 \mathrm{~d}$. This discovery is the 9th hot Jupiter from $K 2$ and highlights $K 2$ 's ability to detect transiting giant planets at periods slightly longer than traditional, ground-based surveys. This planet is slightly inflated, but much less than others with similar incident fluxes. These are of interest for investigating the inflation mechanism of hot Jupiters.
\end{abstract}

Key words: techniques: high angular resolution-techniques: photometric-techniques: radial velocities - planets and satellites: detection-stars: individual: K2-140.

\section{INTRODUCTION}

Transiting exoplanets offer the best insight into worlds outside our Solar system, as we can determine the mass, radius, and obtain information regarding the planetary atmosphere. Traditional groundbased surveys such as HAT-Net (Bakos et al. 2004), WASP (Pollacco et al. 2006) and KELT (Pepper et al. 2007) are predominately sensitive to very short period transiting giant planets $(\mathrm{P} \sim 3 \mathrm{~d})$. Longer period transiting systems have proved much more difficult to detect. Some advantage has been gained using multisite surveys, with HATSouth (Bakos et al. 2013) detecting planets in periods as long as $16 \mathrm{~d}$ (Brahm et al. 2016b). However, the continuous monitoring enabled by space-based telescopes has allowed for a dramatic increase in the number of longer period transiting systems. The Kepler mission (Borucki et al. 2010; Jenkins et al. 2010; Koch et al. 2010), with 4 yr of near-continuous coverage, has uncovered a host of transiting planets with longer periods, however many of these transit stars that are too faint to allow for planetary mass determination via radial velocities. In 2013, after 4 yr of observations, the second of Kepler's 
four reaction wheels failed. From this, the $K 2$ mission was born (Howell et al. 2014). Unlike the original mission, which observed a single region of the sky, $K 2$ observes proposed targets within a series of fields lying along the ecliptic, continuously, for $\sim 80 \mathrm{~d}$. The adverse impact of the two failed reaction wheels has been minimized, but there is now a 6-h roll effect affecting $K 2$ light curves. This causes brightness changes as stars move from pixel to pixel on the $\mathrm{CCD}$. However, there have been many different attempts to calibrate this effect and remove it from the light curves, allowing for transiting exoplanet searches (Vanderburg \& Johnson 2014; Armstrong et al. 2015; Aigrain, Parviainen \& Pope 2016). Further, the continuous observations for $80 \mathrm{~d}$ still allows for longer period systems to be discovered, e.g. EPIC 201702477b (40.736d, Bayliss et al. 2017b). Additionally, a number of more typical hot Jupiters have been discovered, e.g. K2-30b (4.099d), K2-34b (2.996d) (Lillo-Box et al. 2016), and K2-31b (1.258d, Grziwa et al. 2016).

In this paper, we report the discovery of K2-140b, a 6.57-d hot Jupiter on an eccentric orbit. In Section 2, we outline the observations that led to the discovery. In Section 3, we describe the analysis of the data that determined its properties. In Section 4, we discuss the properties and the planet's position with respect to other known hot Jupiters, and in Section 5, we summarize the discovery.

\section{OBSERVATIONS}

In this section, we set out the observations made to detect and characterize the transiting exoplanet K2-140b.

\subsection{K2 Photometry}

The light curve for K2-140 came from Campaign 10 of the $K 2$ mission. This campaign observed 41607 targets in long cadence (30 $\mathrm{min})$ and 138 targets in short cadence $(1 \mathrm{~min})$ in the ecliptic plane centred around RA $12 \mathrm{~h} 27 \mathrm{~m}$ 07.07s Dec. $-04^{\circ} 01^{\prime} 37.77^{\prime \prime}$. Due to a pointing error (targets were off by 12 arcsec, meaning many fell outside their apertures), this campaign was split into two data releases, C10a and C10b. C10a lasted $6 \mathrm{~d}$ between 2016 July 6 19:45:29 UTC and 2016 July 13 01:19:55 UTC. The second release, $\mathrm{C} 10 \mathrm{~b}$, was observed for $69 \mathrm{~d}$. However, there was a data gap of $14 \mathrm{~d}$ after $7 \mathrm{~d}$ of observing due to module 4 of the telescope failing which powered off the photometer.

After the public release of the data on 2016 December 20, the light curves reduced by the $K 2$ Science team were downloaded and analysed for planetary signals (light-curve data listed in Table 2). This analysis required long-term variations to be removed from the light curve. This was done by fitting a sliding polynomial, which fits locally a polynomial to a small section ('step size') of the light curve using a significantly larger section ('window') of the surrounding light curve, and dividing it out. For the sliding polynomial, we used a third-order polynomial with a step size of $0.1 \mathrm{~d}$ and a window size of $5 \mathrm{~d}$. To ensure the result is not jagged, the step size must be significantly smaller than the window size and to ensure that the transit is not accidentally fitted and removed by the process, to ensure the transit is left intact requires outlier rejection from the polynomial fit - this was done with a strict cut of positive outliers and a looser negative outlier cut. To search for planetary transits, we used a PYTHON-wrapped ${ }^{1}$ version of the BLS routine (Kovács, Zucker \& Mazeh 2002) to initially search for any significant signals and then a second time focused on the signal of interest to determine the transit parameters as accurately as possible. We then phase fold and output the light curve for visual inspection. This transit search found many candidates, which included K2-140 - a 6.57-d planet with a 1.26 per cent transit signal (Fig. 1).

Additionally, as can be seen in Fig. 1, there is some evidence of aliasing in the cadence. This is due to the observed rotation period being a half integer multiple of the cadence of $K 2$.

\subsection{Radial velocities}

We observed K2-140 using the CORALIE spectrograph (Queloz et al. 2000) on the 1.2-m Euler Telescope at La Silla Observatory in Chile. CORALIE is a fibre-fed, high resolution $(R=60000)$ echelle spectrograph capable of delivering $<6 \mathrm{~m} \mathrm{~s}^{-1}$ accuracy. Observations were made between 2017 February 20 and 2017 April 8. Additionally, K2-140 was observed using the High Accuracy Radial Velocity Planet Searcher (HARPS, Mayor et al. 2003) mounted on the ESO 3.6-m telescope in La Silla Observatory in Chile, on February 22 and between April 23 and 28. The spectra, which have a resolution $R=115000$, were reduced using the Collection of Elemental Routines for Echelle Spectra (CERES, Brahm, Jordán \& Espinoza 2017a).

The associated errors with each instrument vary significantly. In the case of CORALIE, the initial errors are higher than HARPS primarily because the star is relatively faint. As a test for the errors, we also calculated the root-mean-square of the data points from the fitted model (see Section 3.2) and they were comparable to the measured errors (see Table 1).

The radial velocities are plotted in Fig. 2, along with the bestfitting model determined by the joint fit described in Section 3.2. The radial velocities are also presented in Table 3.

In order to check radial velocity variation induced by a blended spectrum, we computed the bisector slope of the cross-correlation function for each observation in the manner described in Queloz et al. (2001). In Fig. 3, we find no correlation between the bisector slope and the measured radial velocity. If the signal detected was due to a blended eclipsing binary, then we may expect to see a strong correlation between the bisectors and radial velocity measurements. The bisector values are presented with the radial velocities in Table 3.

\subsection{LCO photometry}

In order to refine the ephemeris, check for TTVs, and check for a colour-dependent transit depth (signifying a probable blend), we performed ground-based photometric follow-up using the Las Cumbres Observatory (LCO) 1-m telescope network (Brown et al. 2013). On 2017 March 18, we monitored the transit in the $i$-band using the three LCO 1-m telescopes situated at South Africa Astronomical Observatory at Sutherland, South Africa (Fig. 4). The observations were taken using the 'Sinistro' camera with exposure times of $120 \mathrm{~s}$ and the telescope defocused $(2.0 \mathrm{~mm})$ to avoid saturation and spread the stellar point-spread function over more pixels - reducing the impact of flat-fielding uncertainties. The images were reduced using the standard LCO reduction pipeline (BANZAI), and then aperture photometry was performed using an automated pipeline (Espinoza et al., in preparation). These observations were made as part of a wider LCO Key Project ${ }^{2}$ to characterize transiting planets using the 


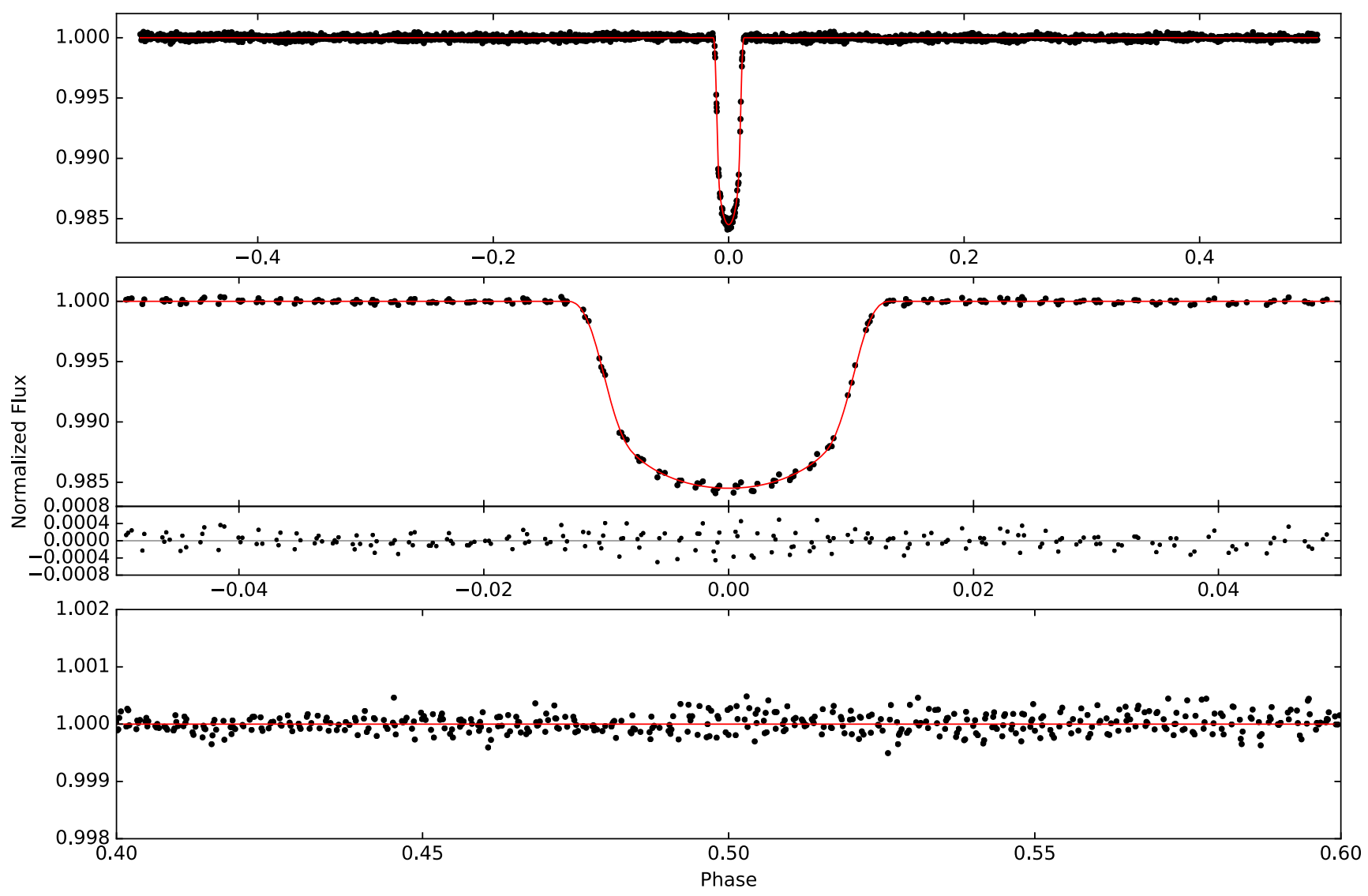

Figure 1. Phase-folded $K 2$ light curve of K2-140 (black points) with best-fitting model plotted as a solid red line (see Section 3.2). Top panel: Full phase light curve with the transit of K2-140b. There are no other significant dips indicating any other transits. Middle panel: Zoom-in of the transit of K2-140b and the resulting residuals from it and the model fit. Bottom panel: Zoom-in around phase 0.5 . There is no indication of an observable secondary eclipse.

LCO 1-m network (see Bayliss et al. 2017b). They are listed in Table 2 .

\subsection{High angular resolution imaging}

High angular resolution imaging of the target was obtained using the Robo-AO instrument (Baranec et al. 2013, 2014) mounted on the Kitt Peak 2.1-m telescope, on the night of 2017 April 15 using the long-pass ' $l p 600$ ' filter (Baranec et al. 2014) with a seeing of $1.5 \mathrm{arcsec}$ and Strehl ratio of 2.7 per cent. The raw rapid read-out data from the Robo-AO visual camera were processed using RoboAO's reduction pipelines described briefly below. A more detailed description can be found in Jensen-Clem et al. (2017).

First, the 'bright-star pipeline generates a windowed data cube centred on an automatically selected guide star. The windowed region is bi-cubically up-sampled and cross correlated with the theoretical PSF to give the centre coordinates of the guide star's PSF in each frame. The nightly dark and dome flat exposures are then used to calibrate the full-frame, unprocessed images. The calibrated full frames are aligned using the centre coordinates identified by the up-sampled, windowed frames, and co-added via the Drizzle algorithm.

Next, the 'high contrast imaging pipeline' generates a 3.5 arcsec frame windowed about the star of interest in the final science frame from the bright star pipeline. A high pass filter is applied to the windowed frame to reduce the contribution of the stellar halo. To whiten correlated speckle noise at small angular separations from the target star, a synthetic PSF generated by the Karhunen-Loève Image Processing (KLIP) algorithm is subtracted from the frame. The KLIP algorithm is based on the method of principal component analysis. The PSF diversity needed to create this synthetic image is provided by a reference library of Robo-AO observations - a technique called Reference star Differential Imaging.

The contrast curve was estimated using the VIP (Vortex Image Processing) package (Gomez Gonzalez et al. 2016) by measuring the residuals from resolution element-sized regions in the PSFsubtracted image.

The final Robo-AO image and contrast curve are shown in Fig. 5. The target is isolated down to $\Delta \mathrm{mag}=4$ at $0.5 \operatorname{arcsec}$ and $\Delta \mathrm{mag}=$ 4.5 at 1 arcsec.

\section{ANALYSIS}

\subsection{Stellar parameters}

Initially, to determine the stellar parameters of K2-140, we built a pipeline for CORALIE spectra based on ISPEC $^{3}$ (Blanco-Cuaresma et al. 2014). This tool provides a large number of options to treat high-resolution spectra (e.g. co-addition, continuum normalization) and it can derive atmospheric parameters and chemical abundances using many different model atmospheres, atomic line lists,

${ }^{3}$ http://www.blancocuaresma.com/s/iSpec 
Table 1. Parameters of K2-140.

\begin{tabular}{|c|c|c|c|}
\hline Parameter & Units & Value & Source \\
\hline EPIC ID & & 228735255 & $\mathrm{H} 16^{a}$ \\
\hline 2MASS ID & & 2MASS J12323296-0936274 & $\mathrm{H} 16^{a}$ \\
\hline $\mathrm{RA}(\alpha)$ & hh:mm:ss & 12: $32: 32.96$ & $\mathrm{H} 16^{a}$ \\
\hline Dec. $(\delta)$ & dd:mm:ss & $-09: 36: 27.5$ & $\mathrm{H} 16^{a}$ \\
\hline g GAIA & mag & 12.393 & $\operatorname{GAIA}^{b}$ \\
\hline$B$ & mag & $13.349 \pm 0.030$ & APASS $^{c}$ \\
\hline$V$ & mag & $12.624 \pm 0.030$ & APASS $^{c}$ \\
\hline$g$ & mag & $12.930 \pm 0.060$ & APASS $^{c}$ \\
\hline$r$ & mag & $12.426 \pm 0.020$ & APASS $^{c}$ \\
\hline$i$ & mag & $12.292 \pm 0.050$ & $\operatorname{APASS}^{c}$ \\
\hline$J$ & mag & $11.421 \pm 0.026$ & $\operatorname{APASS}^{c}$ \\
\hline$H$ & mag & $11.068 \pm 0.021$ & APASS $^{c}$ \\
\hline$K_{\mathrm{S}}$ & mag & $10.995 \pm 0.021$ & $2 \mathrm{MASS}^{d}$ \\
\hline Kep & mag & 12.483 & $\mathrm{H} 16^{a}$ \\
\hline$W_{1}$ & mag & $10.985 \pm 0.024$ & ALLWISE $^{e}$ \\
\hline$W_{2}$ & mag & $11.030 \pm 0.021$ & ALLWISE $^{e}$ \\
\hline$W_{3}$ & mag & $10.891 \pm 0.119$ & ALLWISE $^{e}$ \\
\hline$W_{4}$ & mag & $8.898 \pm-$ & ALLWISE $^{e}$ \\
\hline Distance & $\mathrm{pc}$ & $340.24 \pm 11.58$ & $f$ \\
\hline Age & Gyr & $4.22 \pm 0.95$ & $f$ \\
\hline Spectral type & & G5 & $f$ \\
\hline$M_{V}$ & mag & $4.965_{-0.066}^{+0.069}$ & $f$ \\
\hline$[\mathrm{Fe} / \mathrm{H}]$ & dex & $0.12 \pm 0.045$ & $f$ \\
\hline$T_{\text {eff }}$ & $\mathrm{K}$ & $5654 \pm 55$ & $f$ \\
\hline $\log (g)$ & dex & $4.452_{-0.009}^{+0.010}$ & $f$ \\
\hline$v \sin i$ & $\mathrm{~km} \mathrm{~s}^{-1}$ & $3.8 \pm 0.2$ & $f$ \\
\hline$P_{\text {rot }}$ & days & $16.3 \pm 0.1$ & $f$ \\
\hline$M_{*}$ & $\mathrm{M}_{\odot}$ & $1.005_{-0.020}^{+0.021}$ & $f$ \\
\hline$R_{*}$ & $\mathrm{R}_{\odot}$ & $0.987_{-0.011}^{+0.011}$ & $f$ \\
\hline$\rho_{*}$ & $\rho \odot$ & $1.048 \pm 0.041$ & $f$ \\
\hline$L_{*}$ & $L_{\odot}$ & $0.893_{-0.048}^{+0.049}$ & $f$ \\
\hline$\mu_{1, \mathrm{~K} 2}$ & & $0.341_{-0.079}^{+0.084}$ & $f$ \\
\hline$\mu_{2, \mathrm{~K} 2}$ & & $0.441_{-0.079}^{+0.111}$ & $f$ \\
\hline$\mu_{1, \mathrm{LCO}}$ & & $0.56_{-0.16}^{+0.18}$ & $f$ \\
\hline$\mu_{2, \mathrm{LCO}}$ & & $0.38_{-0.17}^{+0.20}$ & $f$ \\
\hline RV residuals (CORALIE) & $\mathrm{km} \mathrm{s}^{-1}$ & 0.0184 & $f$ \\
\hline RV residuals (HARPS) & $\mathrm{km} \mathrm{s}^{-1}$ & 0.0097 & $f$ \\
\hline
\end{tabular}

${ }^{a}$ Huber et al. (2016), ${ }^{b}$ Gaia Collaboration et al. (2016a,b),

${ }^{c}$ Henden \& Munari (2014), ${ }^{d}$ Skrutskie et al. (2006),

${ }^{e}$ Wright et al. (2010); Mainzer et al. (2011), ${ }^{f}$ This work

radiative transfer codes, and spectroscopic techniques (i.e. equivalent width and synthetic spectral fitting). For this study, we executed the following steps:

(i) Align and co-add all the observations taken with CORALIE (see Section 2.2) to increase the $\mathrm{S} / \mathrm{N}$.

(ii) Reduce the spectrum to the optical wavelength range (480$680 \mathrm{~nm})$.

(iii) Cross-correlate with a solar template to shift the observed spectrum to the rest frame.

(iv) Discard negative fluxes and estimate flux errors based on an estimated S/N.

(v) Convolve to a resolution of $R \sim 47000$ and homogeneously re-sample the spectrum.

(vi) Ignore regions affected by telluric lines.

(vii) Fit the pseudo-continuum and normalize the spectrum.

(viii) Derive atmospheric parameters using the synthetic spectral fitting technique, SPECTRUM (Gray \& Corbally 1994) as radiative transfer code, atomic data obtained from VALD (Kupka, Dubernet \& VAMDC Collaboration 2011), a line selection based on a $R \sim 47000$ solar spectrum (Blanco-Cuaresma et al. 2016, 2017) and the MARCS model atmospheres (Gustafsson et al. 2008).

As an output we obtained the effective temperature, surface gravity $(\log g)$, and metallicity. From these, a series of isochrones were generated using stellar model generator SYCLIST $^{4}$ (Mowlavi et al. 2012). A grid of ages at a given metallicity $(Z=0.040)$ was generated and interpolated to determine the stellar age, mass, radius, and luminosity.

The results of the ISPEC analysis gave an effective temperature of $5732 \pm 32 \mathrm{~K}$, a $\log g$ of $4.29 \mathrm{dex}$ and $[\mathrm{Fe} / \mathrm{H}]=0.32 \pm 0.03 \mathrm{dex}$.

Following a similar procedure, the individual HARPS spectra were median combined in order to construct a higher SNR template.

\footnotetext{
${ }^{4}$ https://obswww.unige.ch/Recherche/evoldb/index/
} 


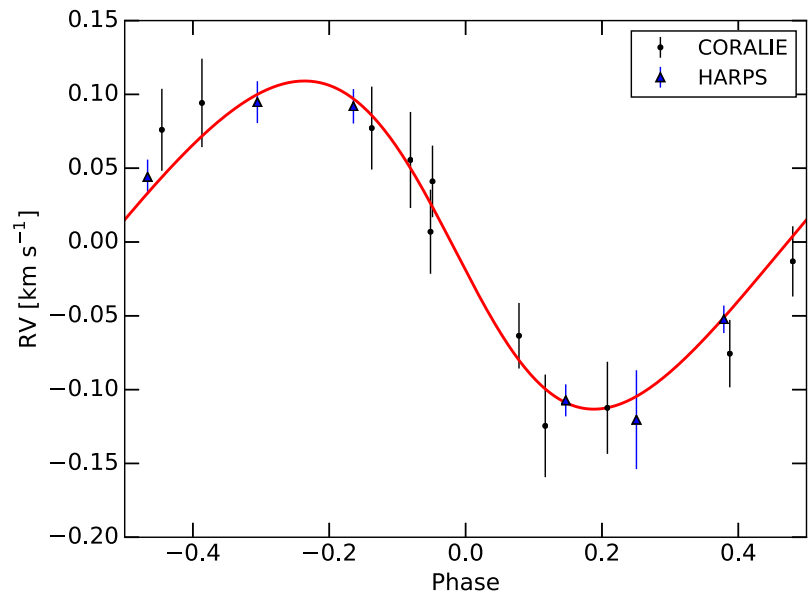

Figure 2. Phase-folded observations from CORALIE (black points) and HARPS (blue triangles) with the best-fitting model (red solid line) as described in Section 3.2. CORALIE observations were taken between 2017 February 20 and 2017 April 8 using the Swiss Euler telescope in La Silla, Chile. HARPS observations were taken between 2017 February 22 and 2017 April 28 using the ESO 3.6-m telescope in La Silla, Chile. The errors for HARPS have had the jitter added in quadrature.

Table 2. Photometry for K2-140.

\begin{tabular}{lcccc}
\hline BJD-2450000 & Flux & Flux error & Filter & Instrument \\
\hline 7582.5906314203 & 1.00002094 & 0.00008399 & kep & K2 \\
7582.6110636177 & 1.00006507 & 0.00008393 & kep & K2 \\
7582.6314957142 & 1.00000389 & 0.00008385 & kep & K2 \\
7582.6519277110 & 1.00001766 & 0.00008376 & kep & K2 \\
7582.6723599071 & 1.00000462 & 0.00008369 & kep & K2 \\
7582.6927920026 & 0.99998520 & 0.00008362 & kep & K2 \\
7582.7132239980 & 1.00001908 & 0.00008351 & kep & K2 \\
7582.7336561931 & 0.99992969 & 0.00008342 & kep & K2 \\
7582.7540882872 & 1.00015249 & 0.00008331 & kep & K2 \\
7582.7745202812 & 0.99993307 & 0.00008323 & kep & K2 \\
- & - & - & - & - \\
\hline
\end{tabular}

${ }^{*}$ Note: partial list - full table available in electronic form.

Table 3. Radial velocities for K2-140b in chronological order.

\begin{tabular}{lllll}
\hline BJD-2450000 & $\begin{array}{c}\text { RV } \\
\mathrm{km} \mathrm{s}^{-1}\end{array}$ & $\begin{array}{l}\text { RV error } \\
\mathrm{km} \mathrm{s}^{-1}\end{array}$ & BIS & Instrument \\
\hline 7804.751722 & 1.25803 & 0.02389 & -0.03886 & CORALIE \\
7806.7167899 & 1.1231 & 0.0333 & -0.019 & HARPS \\
7814.792453 & 1.20384 & 0.02344 & -0.08219 & CORALIE \\
7815.668909 & 1.31119 & 0.02967 & -0.03487 & CORALIE \\
7817.678137 & 1.27255 & 0.03227 & -0.06864 & CORALIE \\
7818.723682 & 1.15348 & 0.02183 & -0.01804 & CORALIE \\
7820.754834 & 1.14137 & 0.02247 & 0.02071 & CORALIE \\
7821.852054 & 1.29300 & 0.02745 & 0.03278 & CORALIE \\
7823.874191 & 1.29414 & 0.02778 & 0.02189 & CORALIE \\
7832.714263 & 1.10465 & 0.03093 & -0.06040 & CORALIE \\
7836.681188 & 1.31122 & 0.03383 & 0.00470 & CORALIE \\
7850.717632 & 1.22394 & 0.02820 & -0.05072 & CORALIE \\
7851.823385 & 1.09243 & 0.03450 & -0.00820 & CORALIE \\
7866.6826602 & 1.1911 & 0.0086 & 0.02 & HARPS \\
7867.699734 & 1.2876 & 0.0111 & -0.009 & HARPS \\
7868.7574426 & 1.3382 & 0.0137 & 0.003 & HARPS \\
7869.6828912 & 1.3354 & 0.0111 & 0.039 & HARPS \\
7871.7285204 & 1.1362 & 0.0102 & 0.0 & HARPS \\
\hline
\end{tabular}

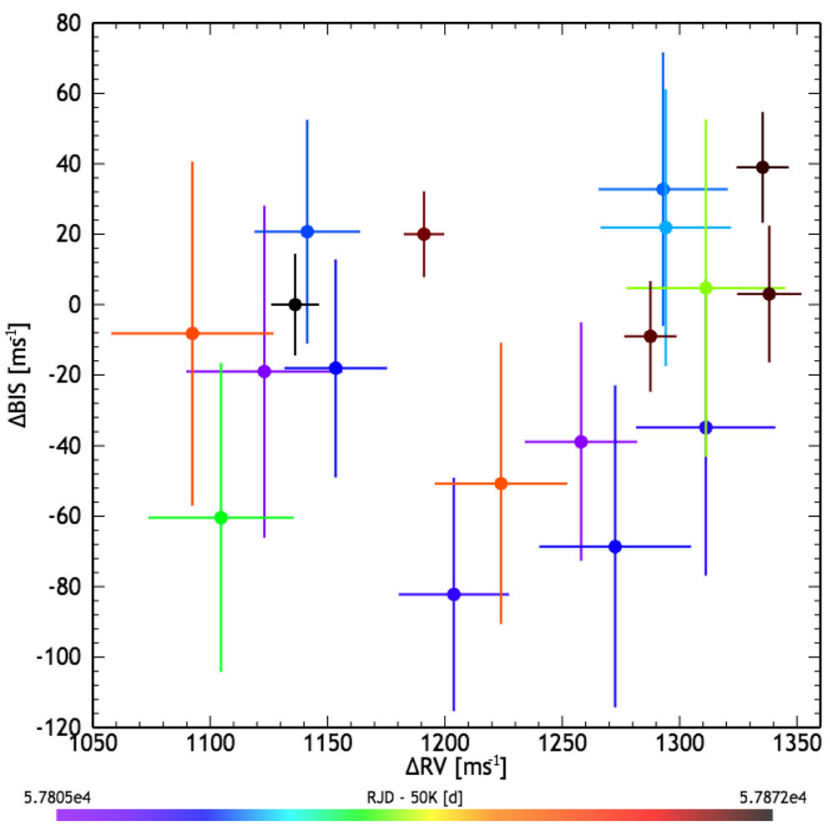

Figure 3. Distribution of the measured radial velocities and associated bisector slopes from CORALIE and HARPS. No evidence of correlation between the two can be seen. Colours represent date of observation between 2017 February 20 and 2017 April 28.

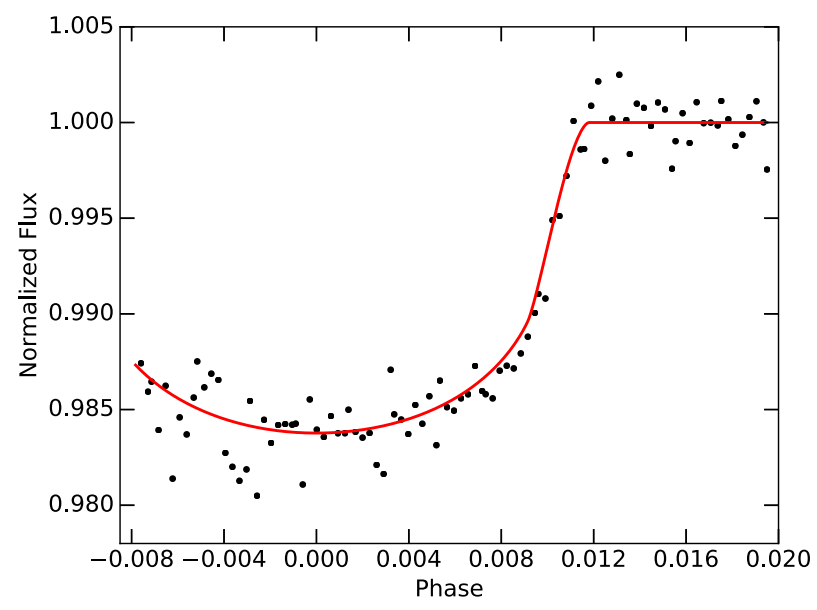

Figure 4. Phase-folded LCO light curve of K2-140 (black points) with best-fitting model plotted as a solid red line (see Section 3.2). Observations occurred the night of 2017 March 18 at the 1-m LCO telescope in Sutherland, South Africa, in ' $i$ ' band.

The resulting spectrum was used as input of the Zonal Atmospheric Parameter estimator (ZASPE, Brahm et al. 2017b) for computing the stellar atmospheric parameters $\left(T_{\mathrm{eff}}, \log g,[\mathrm{Fe} / \mathrm{H}]\right.$ and $\left.v_{\text {rot }} \sin i\right)$ by comparing it with a grid of synthetic spectra generated from the ATLAS9 model atmospheres (Kurucz 1993).

For estimating an initial guess of the physical parameters of the star, we used the Yonsei-Yale Isochrones (Yi et al. 2001) by searching for the $M_{*}$ and stellar Age of the model that would produce the observed $T_{\text {eff }}$ and $a / R_{*}$ values for the given $[\mathrm{Fe} / \mathrm{H}]$. For obtaining the errors in the physical parameters, we performed Monte Carlo simulations where new values for $T_{\text {eff }}, a / R_{*}$, and $[\mathrm{Fe} / \mathrm{H}]$ were sampled from Gaussian distributions in each realization.

The resulting physical parameters were used to compute a more precise value for the stellar $\log g$ than the one obtained from 


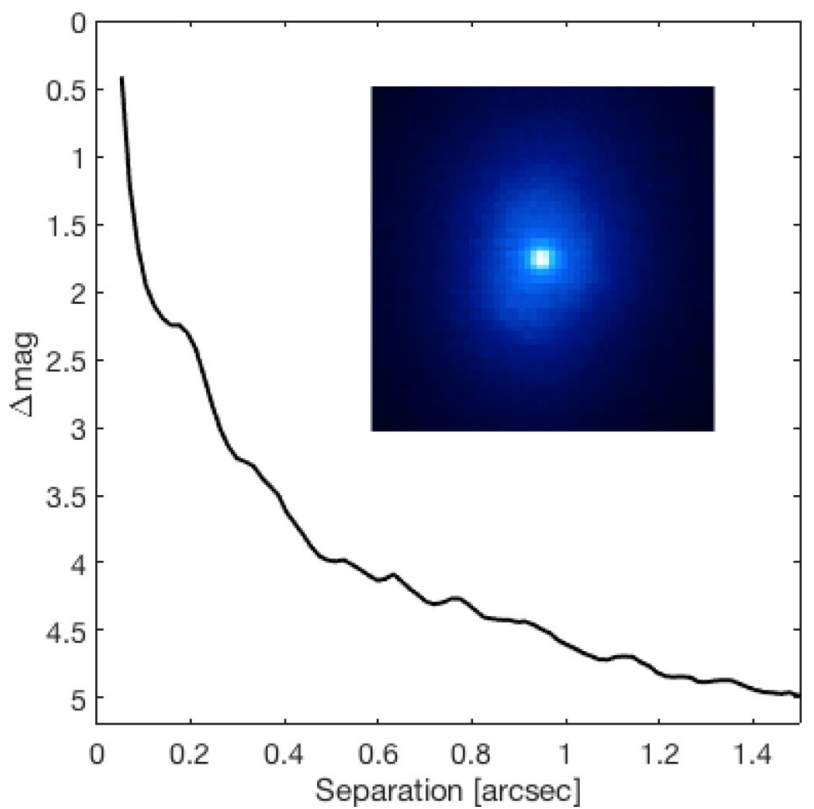

Figure 5. Contrast curve of K2-140 showing the upper limit on the magnitude difference between the target and a possible nearby star as a function of angular separation in arcsec. Data were taken by Robo-AO with the longpass filter $l p 600$ covering a wavelength range from $600 \mathrm{~nm}$ to close to $1 \mu \mathrm{m}$ (Baranec et al. 2014; Jensen-Clem et al. 2017). The inset shows the image of the target spanning 1 arcsec on the side.

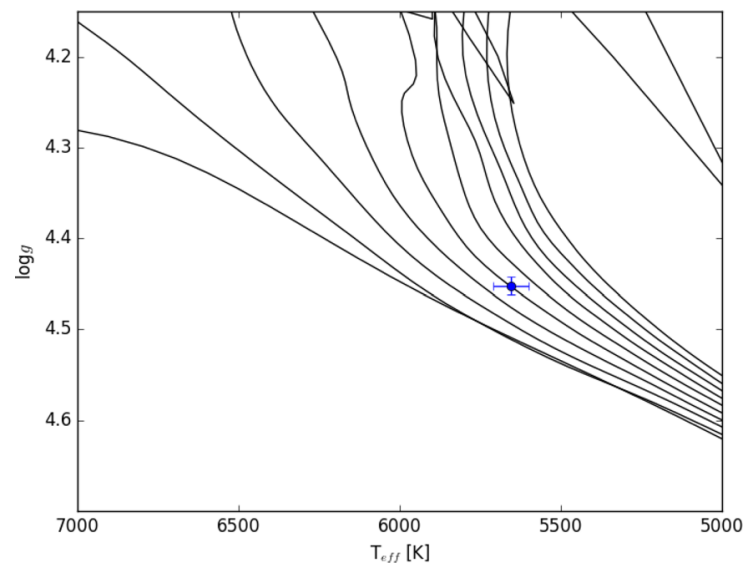

Figure 6. Yonsei-Yale isochrones (Yi et al. 2001) covering (from the lefthand to right-hand side) $0.1,1,2,3,4,5,6,7,8$, and 9 Gyr based on the stellar parameters determined for K2-140.

spectroscopy. The new $\log g$ value was then held fixed in a new ZASPE execution, whose results are displayed in Table 1 . The new atmospheric parameters were used to determine a new set of physical parameters from the Yonsei-Yale Isochrones (Fig. 6), obtaining a stellar mass of $1.005 \pm 0.020 \mathrm{M}_{\odot}$, a stellar age of $4.2 \pm 1.0 \mathrm{Gyr}$, and a corresponding stellar radius of $0.987 \pm 0.011 \mathrm{R}_{\odot}$, making this host star a slightly metal-rich solar analogue.

Comparing the two results (ISPEC and ZASPE), a very similar effective temperature was measured. However, the ISPEC routine detected a smaller $\log g$ and higher metallicity. We attribute this discrepancy to the HARPS data having a significantly better signalto-noise ratio than the reconnaissance spectroscopy, which was measured from CORALIE. For further analysis, the results from ZASPE using HARPS data were used.

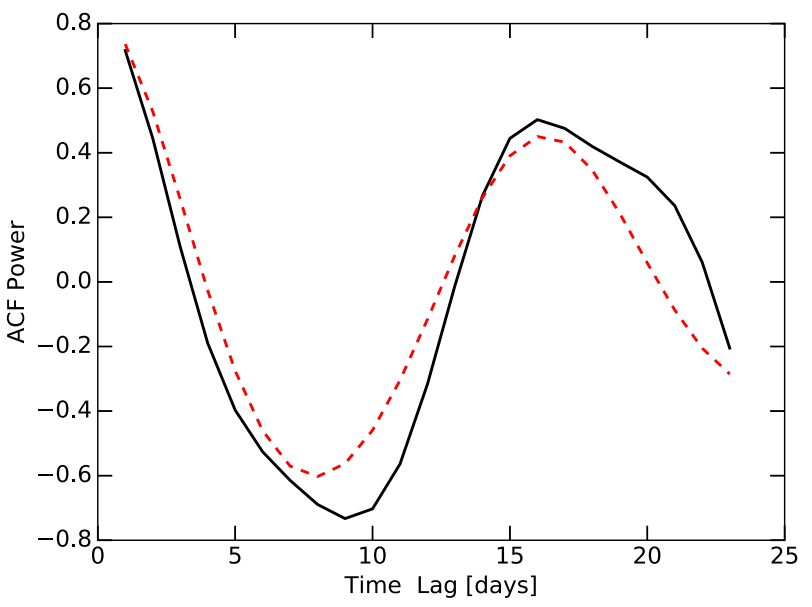

Figure 7. The autocorrelation function (black line) of the light curve for K2-140 with the transits omitted, fitted with a harmonic function (red dashed line) using method described in Giles et al. (2017). This measured a rotation period for K2-140 of $16.3 \pm 0.1 \mathrm{~d}$.

We measured the rotation period of K2-140 using an autocorrelation function of the polynomial fit of the $K 2$ light curve (with the transit omitted) as described in Giles et al. (2017). This determined a rotation period of $16.3 \pm 0.1 \mathrm{~d}$ (Fig. 7). Given $R_{*}=0.987_{-0.011}^{+0.011}$, this rotation should result in a $v \sin i \sim 3.1 \mathrm{~km} \mathrm{~s}^{-1}$, assuming stellar spin axis is perpendicular to the orbital plane of the planet. The spectroscopically derived $v \sin i$ is slightly larger than this value ( $v \sin i=3.8 \pm 0.2$ ), which may be due to non-equatorial spots and solar-like differential rotation. Such an effect has been seen in other $K 2$ transiting systems, e.g. HATS-36b (Bayliss et al. 2017a).

\subsection{Joint fit}

We fit the photometry data from Sections 2.1 and 2.3 jointly with the radial velocities from Section 2.2 using the exonailer ${ }^{5}$ algorithm (Espinoza et al. 2016). The exonailer fitting was conducted with loose priors on the period, $P$, time of first transit, $T_{0}$, and planet-to-stellar-radii ratio, $p$ (see Table 4 for priors used). These were determined directly from the $K 2$ light curve. In addition, extra (Gaussian) noise terms were added to the errors of the LCO and $K 2$ photometry (in order to empirically estimate extra photometric jitter), with a prior of $\mathcal{N}(11000)$ for each. Extra Gaussian noise terms were also added to the CORALIE and HARPS radial velocities (in order to model radial-velocity jitter either instrumental or from stellar origin due to, e.g. activity).

Special care was taken in the modelling of the limb-darkening effect, as it is known that this can have a direct impact on the retrieved fitted transit parameters (Espinoza \& Jordán 2015). In order to select the best limb-darkening law, we followed Espinoza \& Jordán (2016) and ran the $1 \mathrm{~d}$-exosim algorithm, ${ }^{6}$ which gives the mean-square error on each of the retrieved transit parameters for a given limb-darkening law (given the noise, sampling and geometry of the transit). The quadratic law was chosen as it was the law that gave the minimum mean-square error on the planet-to-star radius ratio. For this case, this was the most important transit parameter because it defines the exoplanet's density. Additionally, the

\footnotetext{
${ }^{5}$ https://github.com/nespinoza/exonailer

${ }^{6} \mathrm{https}: / /$ github.com/nespinoza/ld-exosim
} 
Table 4. Parameters of K2-140b.

\begin{tabular}{|c|c|c|c|}
\hline Parameter & Units & Value & Priors $^{a}$ \\
\hline Period & days & $6.569300_{-0.000020}^{+0.000017}$ & $\mathcal{N}(6.569,0.01)$ \\
\hline$T_{0}$ & days & $2457588.28380_{-0.00014}^{+0.00014}$ & $\mathcal{N}(2457588.28544,0.01)$ \\
\hline$T_{14}$ & hours & $4.56 \pm 0.29$ & \\
\hline$T_{23}$ & hours & $3.49 \pm 0.26$ & \\
\hline$T_{12}=34$ & hours & $0.53 \pm 0.19$ & \\
\hline$R_{\mathrm{P}} / R_{*}$ & & $0.1140_{-0.0012}^{+0.0015}$ & $\mathcal{U}(0.05,0.2)$ \\
\hline$b$ & & $0.33 \pm 0.14$ & \\
\hline$i$ & $\circ$ & $88.51_{-0.53}^{+0.69}$ & $\mathcal{U}(80,90)$ \\
\hline$a_{*}$ & $\mathrm{au}$ & $0.0591 \pm 0.0034$ & $\mathcal{U}(3.0,30.0)\left[\mathrm{R}_{*}\right]$ \\
\hline$K$ & $\mathrm{~km} \mathrm{~s}^{-1}$ & $0.1112_{-0.0073}^{+0.0076}$ & $\mathcal{N}(0.1,0.1)$ \\
\hline$\gamma_{\text {CORALIE }}$ & $\mathrm{km} \mathrm{s}^{-1}$ & $1.2170_{-0.0092}^{+0.0089}$ & $\mathcal{N}(1.22,0.05)$ \\
\hline$\gamma_{\text {HARPS }}$ & $\mathrm{km} \mathrm{s}^{-1}$ & $1.2435_{-0.0070}^{+0.0068}$ & $\mathcal{N}(1.24,0.05)$ \\
\hline CORALIE jitter & $\mathrm{km} \mathrm{s}^{-1}$ & $0.0041_{-0.0037}^{+0.0127}$ & $\mathcal{J}(0.0001,0.1)$ \\
\hline HARPS jitter & $\mathrm{km} \mathrm{s}^{-1}$ & $0.0037_{-0.0033}^{+0.0144}$ & $\mathcal{J}(0.0001,0.1)$ \\
\hline Incident flux $\langle F\rangle$ & $10^{8} \mathrm{erg} \mathrm{s}^{-1} \mathrm{~cm}^{-2}$ & $2.565 \pm 0.105$ & \\
\hline $\mathrm{e}$ & & $0.120_{-0.046}^{+0.056}$ & $\beta(0.867,3.03)$ \\
\hline$\omega$ & $\circ$ & $98.88_{-4.16}^{+3.85}$ & $\mathcal{U}(0.0,180.0)$ \\
\hline $\mathrm{M}_{\mathrm{P}}$ & $\mathrm{M}_{\text {Jup }}$ & $1.019 \pm 0.070$ & \\
\hline $\mathrm{R}_{\mathrm{P}}$ & $\mathrm{R}_{\text {Jup }}$ & $1.095 \pm 0.018$ & \\
\hline $\log g_{P}$ & $\operatorname{dex}(\operatorname{cgs})$ & $3.324 \pm 0.033$ & \\
\hline$\rho_{\mathrm{P}}$ & $\rho_{\text {Jup }}$ & $0.726 \pm 0.062$ & \\
\hline$T_{\text {eq. }}$ & $\mathrm{K}$ & $1114 \pm 34$ & \\
\hline
\end{tabular}

$\overline{a_{\mathcal{N}}(\mu, \sigma) \text { is a normal distribution with mean } \mu \text { and standard deviation } \sigma ; \mathcal{U}(a, b) \text { is a uniform distribution }}$ between values $a$ and $b ; \mathcal{J}(a, b)$ is a Jeffrey's distribution with a lower limit of $a$ and $b$; and $\beta(a, b)$ is a Beta distribution with parameters $a$ and $b$ as described by Kipping (2013a).

limb-darkening coefficients were individually fitted for the $K 2$ and LCO light curves, as they have different response functions and span different wavelength ranges. An initial fit assuming different planet-to-star radius ratios for each data set was also made, but both independently gave consistent parameters with no wavelength dependence. The final fit was made by using a common planet-to-star radius ratio for both data sets. Priors for the limb-darkening coefficients were set to be $\mathcal{N}(0,1)$, an uninformative transformation of the quadratic limb-darkening parameters (see Kipping 2013b).

We tried fits assuming circular and non-circular orbits and the results favour the non-circular orbit, which gave an eccentricity of $0.120_{-0.046}^{+0.056}$ and an argument of periapsis of $98.88_{-4.16}^{+3.85}$.

The final fits for the $K 2$ and LCO light curves are shown in red in Figs 1 and 4, and for the radial-velocities in Fig. 2. The priors and posterior values of the fitted parameters with exonailer are listed in Table 4. As can be seen, the photometric jitter is significant only for the $K 2$ light curve; the LCO photometric jitter is consistent with zero. This is due to the fact that we decided to estimate the errors directly from the $K 2$ photometry, whereas the extra jitter was added in quadrature to the LCO errorbars given by the photometric pipeline. For the radial-velocity jitter, it can be seen that the extra term for both instruments is also consistent with zero.

\subsection{Planet parameters}

exona i ler was able to determine various system parameters from the light curve transit shape: $a / R_{*}$, the semi-major axis-to-stellar radius ratio; $\mathrm{R}_{p} / \mathrm{R}_{*}$, the ratio of planetary to stellar radius (given as $p$ in exonailer); $\mathrm{t}_{0}$, the time of the first observed transit; $P$, the orbital period of the planet; and $i$, the inclination of the planet's orbit. Additionally, from the radial velocity curves: $e$, the eccentricity; $\omega$, the periapsis argument; and $K$, the radial velocity semi-amplitude of the star. Through a combination of these parameters and the already determined stellar mass and radius from Section 3.1, further properties of the planet can be determined using the equations as described in Seager \& Mallén-Ornelas (2003). We measured the planetary mass to be $1.019 \pm 0.070 \mathrm{M}_{\text {Jup }}$ with a radius of $1.095 \pm 0.018 \mathrm{R}_{\mathrm{Jup}}$. This indicates a bulk density which is slightly less than that of Jupiter, $0.726 \pm 0.062 \rho_{\text {Jup }}$. The planet has an incident flux of $2.565 \pm 0.105 \times 10^{8} \mathrm{erg} \mathrm{s}^{-1} \mathrm{~cm}^{-2}$. The predicted equilibrium temperature is $1114 \pm 34 \mathrm{~K}$, with the assumption of a blackbody and an efficient transfer of energy from the day- to night-side. These are all listed in Table 4.

\section{DISCUSSION}

In this section, we compare the properties of K2-140b to the population of known hot Jupiters, and for this purpose, we use the NASA Exoplanet Archive $^{7}$ (Akeson et al. 2013) as accessed on 2017 June 6.

\subsection{Orbital period}

For warm and hot Jupiters, Kepler dominates the number of discoveries for planets with orbital periods equivalent to or longer than that of K2-140b. Therefore, to investigate the ability of $K 2$ to find longer period warm and hot Jupiters, we compare the $\mathrm{Ke}$ pler and $K 2$ discoveries within a subset of all confirmed planets. They all have masses greater than $0.2 \mathrm{M}_{\mathrm{Jup}}$ and have other significant parameters - such as eccentricity, planet density, and planet

${ }^{7}$ exoplanetarchive.ipac.caltech.edu 
Table 5. $K 2$ discovered warm and hot Jupiters with precise measurements ( 20 per cent) on the masses and radii.

\begin{tabular}{lcccc}
\hline Planet & $P_{\text {orb }}(\mathrm{d})$ & Mass $\left(\mathrm{M}_{\text {Jup }}\right)$ & Radius $\left(\mathrm{R}_{\text {Jup }}\right)$ & Reference \\
\hline K2-29b & $3.2588321 \pm 0.0000019$ & $0.73 \pm 0.04$ & $1.19 \pm 0.02$ & Santerne et al. (2016) \\
$"$ & $3.2589263 \pm 0.0000015$ & $0.613_{-0.026}^{+0.027}$ & $1.000_{-0.067}^{+0.071}$ & Johnson et al. (2016) \\
K2-30b & $4.098503 \pm 0.000011$ & $0.579_{-0.027}^{+0.028}$ & $1.039_{-0.051}^{+0.050}$ & Johnson et al. (2016) \\
$"$ & $4.098513 \pm 0.000018$ & $0.625 \pm 0.030$ & $1.197 \pm 0.052$ & Lillo-Box et al. (2016) \\
K2-31b & $4.09849_{-0.00002}^{+0.0002}$ & $0.589_{-0.022}^{+0.023}$ & $1.069_{-0.019}^{+0.023}$ & Brahm et al. (2016a) \\
K2-34b & $1.257850 \pm 0.000002$ & $1.774 \pm 0.079$ & $0.71-1.41$ & Grziwa et al. (2016) \\
$"$ & $2.9956675_{-0.000071}^{+0.000075}$ & $1.649 \pm 0.098$ & $1.217 \pm 0.053$ & Lillo-Box et al. (2016) \\
K2-60b & $2.995654 \pm 0.000018$ & $1.773 \pm 0.086$ & $1.44 \pm 0.16$ & Hirano et al. (2016) \\
K2-97b & $2.995629_{-0.0000006}^{+0.00006}$ & $1.698_{-0.050}^{+0.061}$ & $1.377_{-0.13}^{+0.14}$ & Brahm et al. (2016a) \\
K2-99b & $3.00265 \pm 0.00004$ & $0.426 \pm 0.037$ & $0.683 \pm 0.037$ & Eigmüller et al. (2017) \\
K2-107b & $8.4016 \pm 0.0015$ & $1.10 \pm 0.11$ & $1.31 \pm 0.11$ & Grunblatt et al. (2016) \\
\hline
\end{tabular}

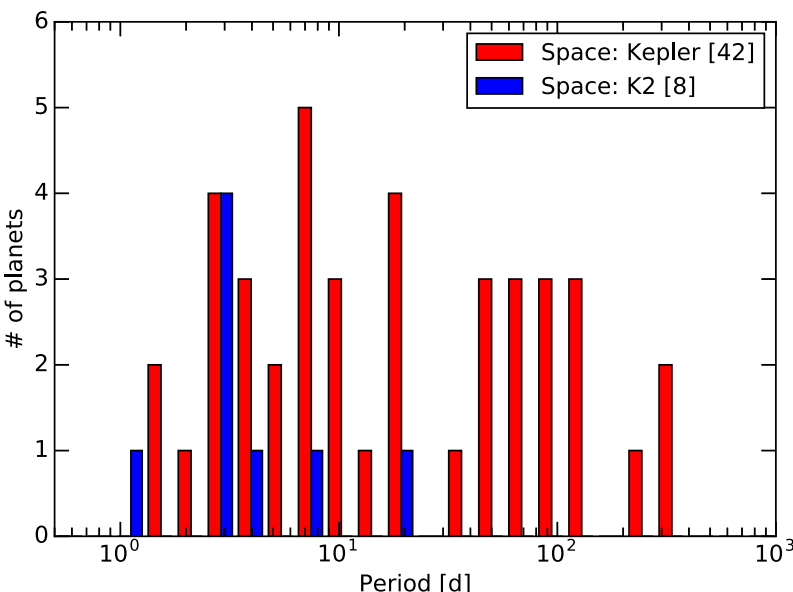

Figure 8. Distribution of confirmed planets found with Kepler and $K 2$ with masses $>0.2 \mathrm{M}_{\text {Jup }}$. Kepler is in red and $K 2$ is in blue. $\mathrm{K} 2-140 \mathrm{~b}$ has been included into the $K 2$ distribution.

radius - also measured. Currently, $K 2$ has only discovered eight planets within this subset where K2-140b is the third longest orbital period (see Table 5).

The number of planets discovered by Kepler and $K 2$ over the period range strongly reveals that, as expected, $K 2$ is less sensitive to long-period planets than Kepler, equally sensitive to short-period planets (Fig. 8). Kepler observed 156000 stars and, to date, $K 2$ has observed a total of 171610 (all stars observed by $K 2$ in long cadence from Campaigns 1-10). However, there will be a natural ramping down of the detection efficiency for planets with periods of $\sim 30$ $40 \mathrm{~d}$ for $K 2$ as campaigns typically do not last longer than $80 \mathrm{~d}-$ whereas, for Kepler, there was almost $4 \mathrm{yr}$ of continuous observation of the survey. Additionally, due to the necessary follow-up time required per planet (radial velocity, imaging etc.), the community has had much longer to confirm Kepler candidates compared with $K 2$ candidates - there are still regular announcements of discoveries from older $K 2$ campaigns as well as discoveries from the current campaign. Given more time, the distribution for planets with orbital periods of $40 \mathrm{~d}$ or less in $K 2$ may reach a similar distribution to Kepler. By the conclusion of the $K 2$ mission (assuming 19 full

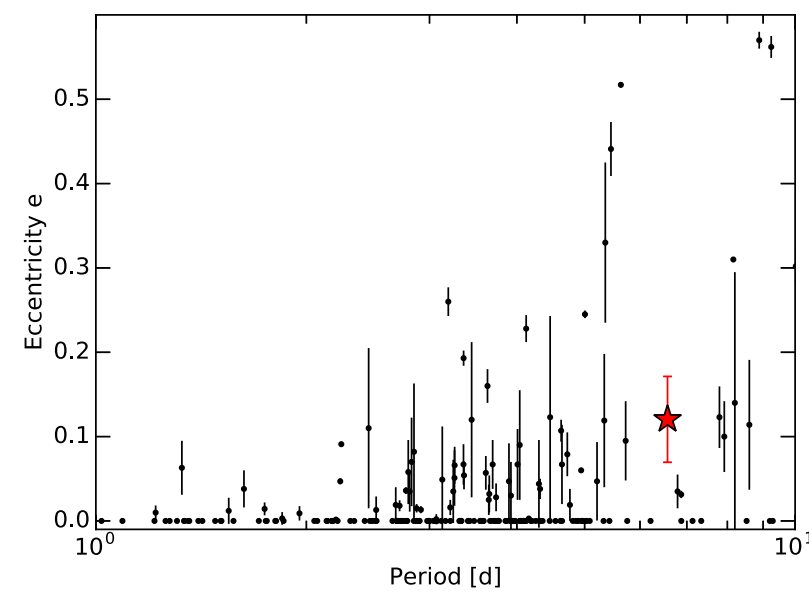

Figure 9. Eccentricities of transiting hot Jupiters $(P=1-10 \mathrm{~d}$, $\left.M_{P}>0.2 \mathrm{M}_{\mathrm{Jup}}\right) . \mathrm{K} 2-140 \mathrm{~b}$ is plotted as a red star. Planets with undetermined eccentricities have been excluded.

campaigns), we may expect $K 2$ will produce more than double the number of transiting giant planets with periods $<10 \mathrm{~d}$ compared to Kepler.

Within the ranges of warm and hot Jupiters, ground-based surveys, in fact, dominate for shorter orbital periods. There are only 11 ground-based discoveries (NASA Exoplanet Archive, Akeson et al. 2013) with periods longer than that of K2-140b. This shows that, compared to ground-based surveys, $K 2$ is more effective at detecting longer period warm and hot Jupiters.

\subsection{Eccentricity}

Exoplanets that have non-zero eccentricities are bodies that have typically either been excited out of their orbits by other bodies or are migrating to a new orbit. The eccentric orbit of K2-140b has the potential to increase our understanding of these mechanisms.

In Fig. 9, we plot the measured eccentricities for transiting hot Jupiters with periods between 1 and $10 \mathrm{~d}$. This was a subset of all confirmed planets with masses greater than $0.2 \mathrm{M}_{\mathrm{Jup}}$ (and as previously, has other significant parameters measured). Below 


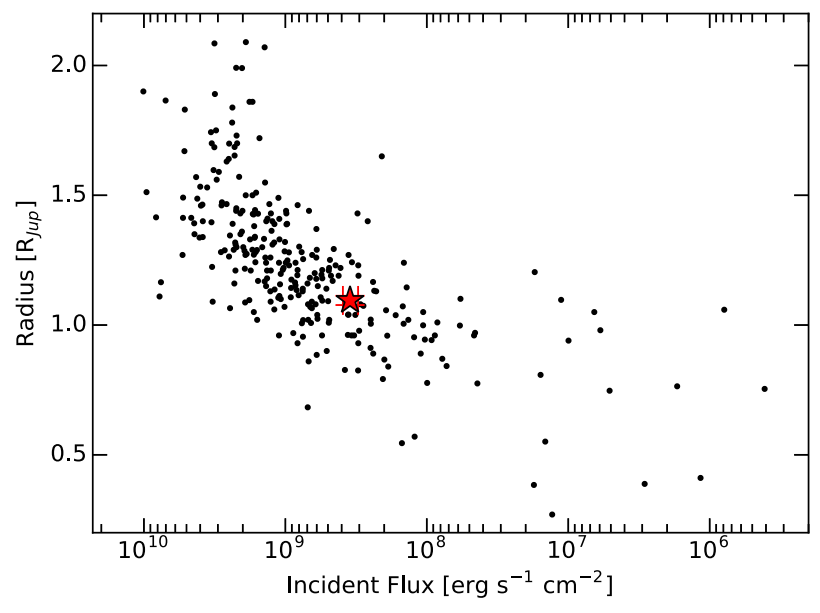

Figure 10. Distribution of incident flux and planet radii of confirmed planets found with measured masses (masses greater than $0.2 \mathrm{M}_{\mathrm{Jup}}$ ) and other measured properties. This work is represented by the red star and all other warm, hot Jupiters by black points.

$\sim 5.5 \mathrm{~d}$, approximately 70 per cent of hot Jupiters have measured eccentricity consistent with 0 . However for systems with period greater than 5.5 , this fraction drops below 50 percent. It is therefore not surprising that we find a non-zero eccentricity for K2-140b $\left(e=0.120_{-0.046}^{+0.056}\right)$. If we assume a Q-factor of $10^{6}(\mathrm{Wu} 2005)$, we calculate (Goldreich \& Soter 1966) a tidal circularization time-scale of $\tau_{e}=2.577$ Gyr. Given our best estimate for the age of the system $(4.22 \pm 0.95 \mathrm{Gyr})$, this means that the time-scale is of the same order of the age of the star.

\subsection{Planet atmosphere inflation}

A common avenue of investigation associated with warm and hot Jupiters is determining whether they are inflated or not. The distribution of the incident flux on a planet and its radii for warm and hot Jupiters is from a subset of confirmed planets with masses greater than $0.2 \mathrm{M}_{\mathrm{Jup}}$ and with other significant parameters measured. Based on the mass and radius of K2-140b, it is slightly inflated compared with Jupiter, but not inflated with respect to other exoplanets with similar incident flux (see Fig. 10). The planet receives an incident flux of $2.565 \pm 0.105 \times 10^{8} \mathrm{erg} \mathrm{s}^{-1} \mathrm{~cm}^{-2}$, which is very close to the empirical limit for inflation $\left(2 \times 10^{8} \mathrm{erg} \mathrm{s}^{-1} \mathrm{~cm}^{-2}\right.$ Demory \& Seager 2011). Discovering exoplanet in this incident flux regime is important for studying the onset of the mechanism by which hot Jupiters are inflated.

\section{CONCLUSIONS}

We found a hot-Jupiter planet in data from K2 Campaign 10 and followed it up with radial velocity measurements and high angular resolution imaging. K2-140b orbits a $\mathrm{V}=12.624 \pm 0.030$, $4.22 \pm 0.95 \mathrm{Gyr}$ star with a $[\mathrm{Fe} / \mathrm{H}]$ of $0.12 \pm 0.045$. The planet has a non-circular orbit with an eccentricity of $0.120_{-0.046}^{+0.056}$ and period of $6.57 \mathrm{~d}$ and a mass and radius of $1.019 \pm 0.070 \mathrm{M}_{\text {Jup }}$ and $1.095 \pm 0.018 \mathrm{R}_{\text {Jup }}$, respectively. It is the third longest period giant exoplanet discovered from $K 2$ and has a period longer period than 94 percent of giant planets discovered from ground-based transit surveys.

\section{ACKNOWLEDGEMENTS}

HACG, DB, CL, SU, FB and MM thank the Swiss National Science Foundation (SNSF) and the Geneva University for their continuous support to our planet search programs. This work has been in particular carried out in the frame of the National Centre for Competence in Research 'PlanetS' supported by the Swiss National Science Foundation (SNSF).

NE acknowledges support from Financiamiento Basal PFB06. $\mathrm{RB}, \mathrm{NE}$, and AJ acknowledge support from the Ministry for the Economy, Development and Tourism Programa Iniciativa Científica Milenio through grant IC 120009, awarded to the Millenium Institute of Astrophysics. AJ acknowledges support by Fondecyt grant 1171208 and partial support by CATA-Basal (PB06, CONICYT). JSJ acknowledges support by Fondecyt grant 1161218 and partial support by CATA-Basal (PB06, CONICYT).

This work has made use of data from the European Space Agency (ESA) mission Gaia (https://www.cosmos.esa.int/gaia), processed by the Gaia Data Processing and Analysis Consortium (DPAC, https://www.cosmos.esa.int/web/gaia/dpac/consortium). Funding for the DPAC has been provided by national institutions, in particular the institutions participating in the Gaia Multilateral Agreement.

This research was made possible through the use of the AAVSO Photometric All-Sky Survey (APASS), funded by the Robert Martin Ayers Sciences Fund.

This publication makes use of data products from the Two Micron All Sky Survey, which is a joint project of the University of Massachusetts and the Infrared Processing and Analysis Center/California Institute of Technology, funded by the National Aeronautics and Space Administration and the National Science Foundation.

Some/all of the data presented in this paper were obtained from the Mikulski Archive for Space Telescopes (MAST). STScI is operated by the Association of Universities for Research in Astronomy, Inc., under NASA contract NAS5-26555. Support for MAST for non-HST data is provided by the NASA Office of Space Science via grant NNX09AF08G and by other grants and contracts.

This paper includes data collected by the Kepler mission. Funding for the Kepler mission is provided by the NASA Science Mission directorate.

This research has made use of the NASA Exoplanet Archive, which is operated by the California Institute of Technology, under contract with the National Aeronautics and Space Administration under the Exoplanet Exploration Program.

This work makes use of observations from the LCO network.

The Robo-AO team thanks NSF and NOAO for making the Kitt Peak 2.1-m telescope available. We thank the observatory staff at Kitt Peak for their efforts to assist Robo-AO KP operations. Robo-AO KP is a partnership between the California Institute of Technology, the University of Hawai' $i$, the University of North Carolina at Chapel Hill, the Inter-University Centre for Astronomy and Astrophysics (IUCAA) at Pune, India, and the National Central University, Taiwan. The Murty family feels very happy to have added a small value to this important project. Robo-AO $\mathrm{KP}$ is also supported by grants from the John Templeton Foundation and the Mt. Cuba Astronomical Foundation. The Robo-AO instrument was developed with support from the National Science Foundation under grants AST-0906060, AST-0960343, and AST1207891, IUCAA, the Mt. Cuba Astronomical Foundation, and by a gift from Samuel Oschin. These data are based on observations at Kitt Peak National Observatory, National Optical Astronomy Observatory (NOAO Prop. ID: 15B-3001), which is operated by the 
Association of Universities for Research in Astronomy (AURA) under cooperative agreement with the National Science Foundation. $\mathrm{CB}$ acknowledges support from the Alfred P. Sloan Foundation. ACC acknowledges support from STFC consolidated grant number ST/M001296/1. DA acknowledges support from STFC consolidated grant reference ST/P000495/1.

This research has made use of the SIMBAD data base and of the VizieR catalogue access tool operated at CDS, France, and used the DACE platform developed in the frame of PlanetS (https://dace.unige.ch).

\section{REFERENCES}

Aigrain S., Parviainen H., Pope B. J. S., 2016, MNRAS, 459, 2408

Akeson R. L. et al., 2013, PASP, 125, 989

Armstrong D. J. et al., 2015, A\&A, 579, A19

Bakos G. Á. et al., 2013, PASP, 125, 154

Bakos G., Noyes R. W., Kovács G., Stanek K. Z., Sasselov D. D., Domsa I., 2004, PASP, 116, 266

Baranec C. et al., 2013, J. Visualized Exp., 72

Baranec C. et al., 2014, ApJ, 790, L8

Bayliss D. et al., 2017a, preprint (arXiv:1706.03858)

Bayliss D. et al., 2017b, AJ, 153, 15

Blanco-Cuaresma S., Soubiran C., Heiter U., Jofré P., 2014, A\&A, 569, A111

Blanco-Cuaresma S. et al., 2016, How much can we trust high-resolution spectroscopic stellar atmospheric parameters? Zenodo

Blanco-Cuaresma S. et al., 2017, in Arribas S., Alonso-Herrero A., Figueras F., Hernández-Monteagudo C., Sánchez-Lavega A., Pérez-Hoyos S., eds, Highlights on Spanish Astrophysics IX. p. 334

Borucki W. J. et al., 2010, Science, 327, 977

Brahm R. et al., 2016a, PASP, 128, 124402

Brahm R. et al., 2016b, AJ, 151, 89

Brahm R., Jordán A., Espinoza N., 2017a, PASP, 129, 034002

Brahm R., Jordán A., Hartman J., Bakos G., 2017b, MNRAS, 467, 971

Brown T. M. et al., 2013, PASP, 125, 1031

Demory B.-O., Seager S., 2011, ApJS, 197, 12

Eigmüller P. et al., 2017, AJ, 153, 130

Espinoza N., Jordán A., 2015, MNRAS, 450, 1879

Espinoza N., Jordán A., 2016, MNRAS, 457, 3573

Espinoza N. et al., 2016, ApJ, 830, 43

Gaia Collaboration et al., 2016a, A\&A, 595, A1

Gaia Collaboration et al., 2016b, A\&A, 595, A2

Giles H., Collier Cameron A., Haywood R., 2017, MNRAS, 472, 1618

Goldreich P., Soter S., 1966, Icarus, 5, 375

Gomez Gonzalez C. A., Absil O., Absil P.-A., Van Droogenbroeck M., Mawet D., Surdej J., 2016, A\&A, 589, A54

Gray R. O., Corbally C. J., 1994, AJ, 107, 742

Grunblatt S. K. et al., 2016, AJ, 152, 185

Grziwa S. et al., 2016, AJ, 152, 132
Gustafsson B., Edvardsson B., Eriksson K., Jørgensen U. G., Nordlund Å., Plez B., 2008, A\&A, 486, 951

Henden A., Munari U., 2014, Contrib. Astron. Obs. Skalnate Pleso, 43, 518

Hirano T. et al., 2016, ApJ, 825, 53

Howell S. B. et al., 2014, PASP, 126, 398

Huber D. et al., 2016, ApJS, 224, 2

Jenkins J. M. et al., 2010, ApJ, 713, L87

Jensen-Clem R., Duev D. A., Riddle R., Salama M., Baranec C., Law N. M., Kulkarni S. R., Ramprakash A. N., 2017, AJ, 155, 32

Johnson M. C. et al., 2016, AJ, 151, 171

Kipping D. M., 2013a, MNRAS, 434, L51

Kipping D. M., 2013b, MNRAS, 435, 2152

Koch D. G. et al., 2010, ApJ, 713, L79

Kovács G., Zucker S., Mazeh T., 2002, A\&A, 391, 369

Kupka F., Dubernet M.-L., VAMDC Collaboration, 2011, Baltic Astron., 20,503

Kurucz R. L., 1993, VizieR Online Data Catalog, 6039

Lillo-Box J. et al., 2016, A\&A, 594, A50

Mainzer A. et al., 2011, ApJ, 731, 53

Mayor M. et al., 2003, The Messenger, 114, 20

Mowlavi N., Eggenberger P., Meynet G., Ekström S., Georgy C., Maeder A., Charbonnel C., Eyer L., 2012, A\&A, 541, A41

Pepper J. et al., 2007, PASP, 119, 923

Pollacco D. L. et al., 2006, PASP, 118, 1407

Queloz D. et al., 2000, A\&A, 354, 99

Queloz D. et al., 2001, A\&A, 379, 279

Santerne A. et al., 2016, ApJ, 824, 55

Seager S., Mallén-Ornelas G., 2003, ApJ, 585, 1038

Skrutskie M. F. et al., 2006, AJ, 131, 1163

Smith A. M. S. et al., 2017, MNRAS, 464, 2708

Vanderburg A., Johnson J. A., 2014, PASP, 126, 948

Wright E. L. et al., 2010, AJ, 140, 1868

Wu Y., 2005, ApJ, 635, 688

Yi S., Demarque P., Kim Y.-C., Lee Y.-W., Ree C. H., Lejeune T., Barnes S., 2001, ApJS, 136, 417

\section{SUPPORTING INFORMATION}

Supplementary data are available at MNRAS online.

Table 2. Photometry for EPIC 228735255.

Please note: Oxford University Press is not responsible for the content or functionality of any supporting materials supplied by the authors. Any queries (other than missing material) should be directed to the corresponding author for the article.

This paper has been typeset from a $\mathrm{T}_{\mathrm{E}} \mathrm{X} / \mathrm{L} \mathrm{T} \mathrm{E}$ file prepared by the author. 